\title{
Remote Access for Fully Automatic Multi-Input Multi-Output Water Level Control System Using SCCT in LabVIEW Environment
}

\author{
Vishal Vasistha \\ (Mechanical, G.L.Bajaj Group of Institutions, Mathura, India)
}

\begin{abstract}
Now a day's experiments/labs remote access via virtual instrumentation concept is very much appreciated due to time saving, without experimental set-up availability and remotely accessed. I have created remote access for my experimental set-up (MIMO water level control system in LABVIEW environment) with the help of SCCT (Smartphone \& Cross-platform Communication Toolkit) platform on LABVIEW software. It's fully automatic and can be accessed and controlled by subscriber any time anywhere.
\end{abstract}

Keywords: Control system, Coupled Tank, LabVIEW, MIMO, SCCT etc.

\section{Introduction}

SCCT (Smartphone \& Cross-platform Communication Toolkit) platform on LABVIEW software is used for experiments communication between publisher and subscriber. Publisher has the full control on remotely accessed experiment by subscriber that how much control he is giving in the hand of subscriber. I have made the Mechatronics experimental setup on water level control system in coupled tank dynamic system in LabVIEW environment. Use of SCCT provides time saving, hands on experiment without setup availability, remotely access etc.

\section{Experimental Setup}

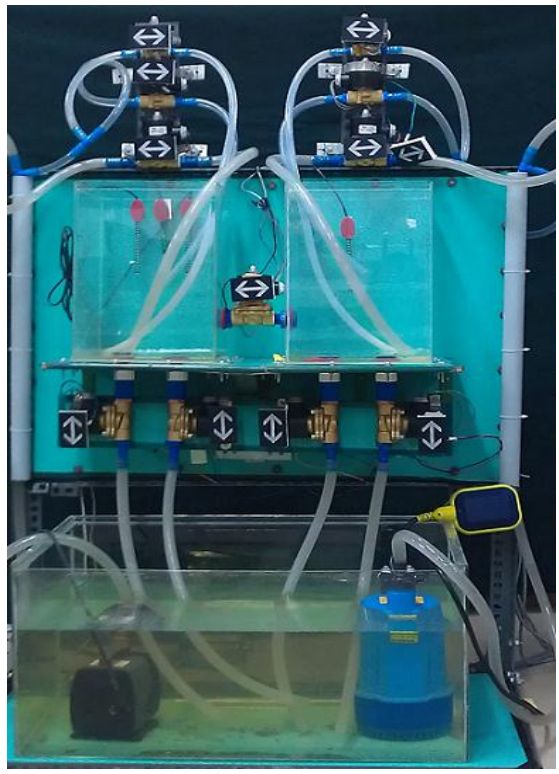

Fig.1- Experimental Set-up

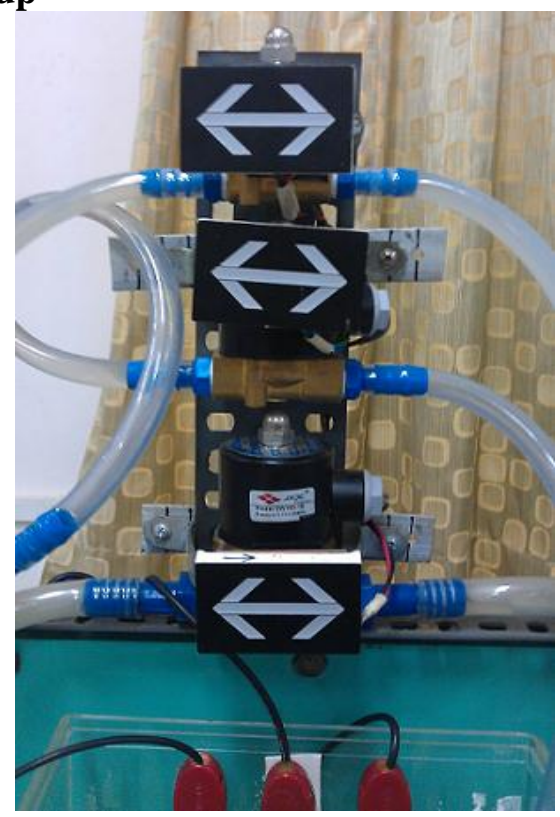

Fig.2 - Solenoid valves installation for input flow

Experimental set-up has been shown in figure -2.1 and 2.2. Arrow LED's have been placed everywhere to show the direction of water flow. Coupled LED between tanks is automatically switched ON/OFF for flow of water as desired water level (set point) is maintained in both the tanks. Solenoid valves placed on top are used for inflow rate and bottom valves as disturbances. Capacitive moisture level sensors have been placed to sense the changing water level in both the tanks and give the reading in form of electrical signal between 0-3 V. Individual pumps have been used for water flow rate in both the tanks. 


\subsection{FRONT PANEL}

\section{Program Coding}

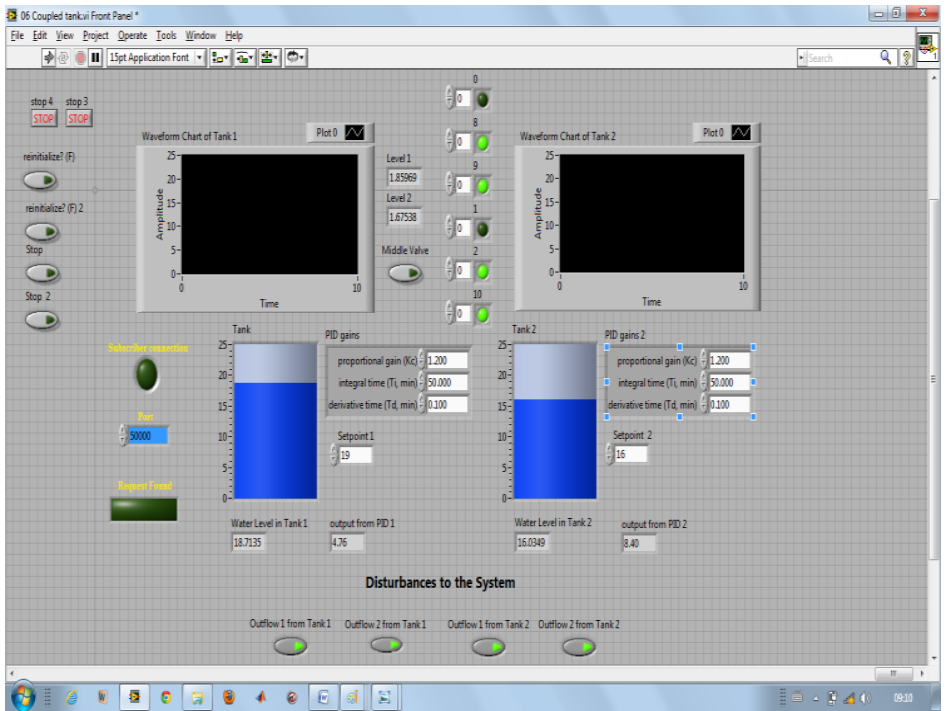

Fig. 3- Front panel for the experiment

Front panel for the experiment has been shown in above figure 3.1. PID gains have been written for both the tanks. Desired water level (SP) has been shown here in the form of waveform chart and virtual tanks. Disturbances (shown down) can be controlled by publisher as well as subscriber. Flexibility has been provided to stop the experiment whenever required may be due to some problem or by wish of subscriber.

\subsection{BLOCK DIAGRAMS}

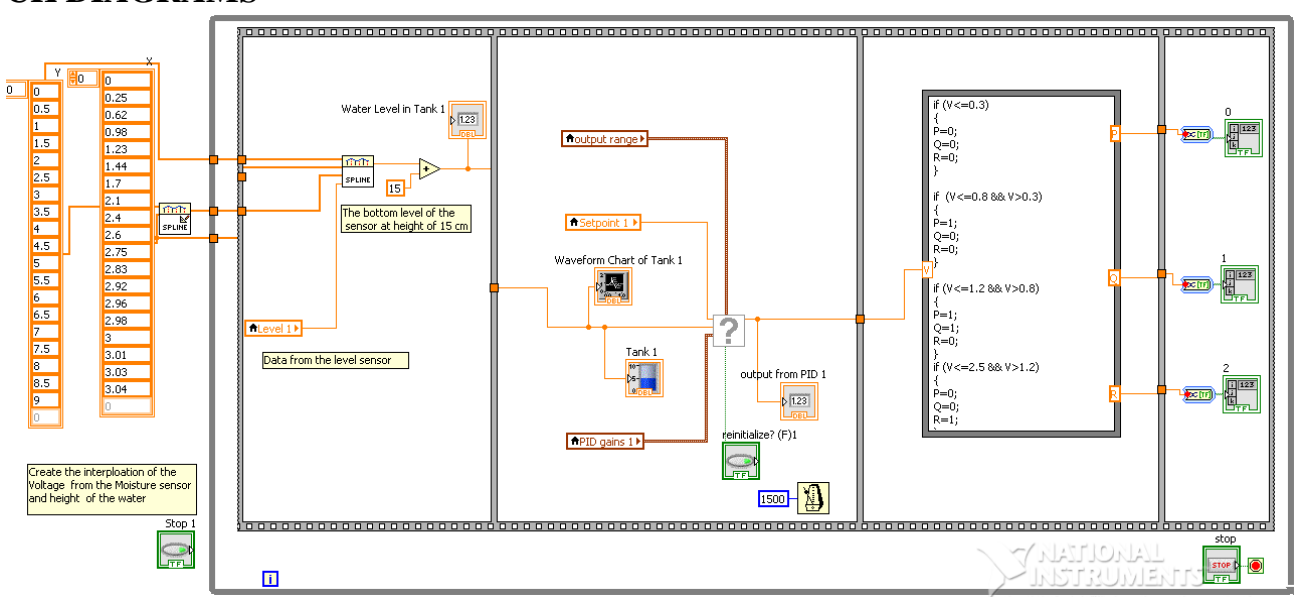

Fig.4 - Block diagram for tank-1 


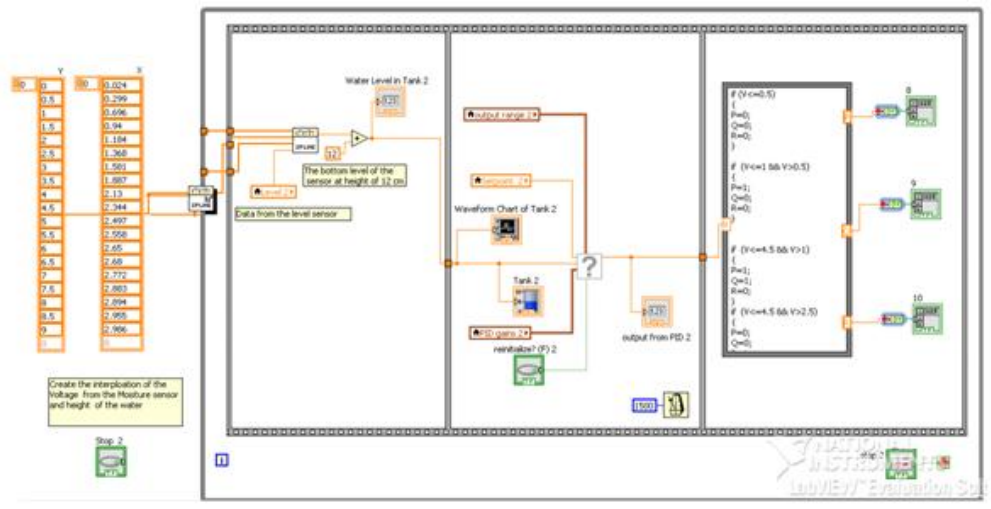

Fig.4 - Block diagram for tank-2

\section{- Represents PID Controller icon}

Above both the block diagrams are similar for tank-1 and tank-2. Data from moisture level sensor has been calibrated in terms of voltage and height. Output form sensor is compared with desired level and controlled by PID controller. All necessary data has been filled in PID block such as set point, PID gains etc. Then PID output has been fuzzified by a particular logic for solenoid valves combinations that has been written in math script ahead of it, to maintain the desired water level in both coupled tanks.
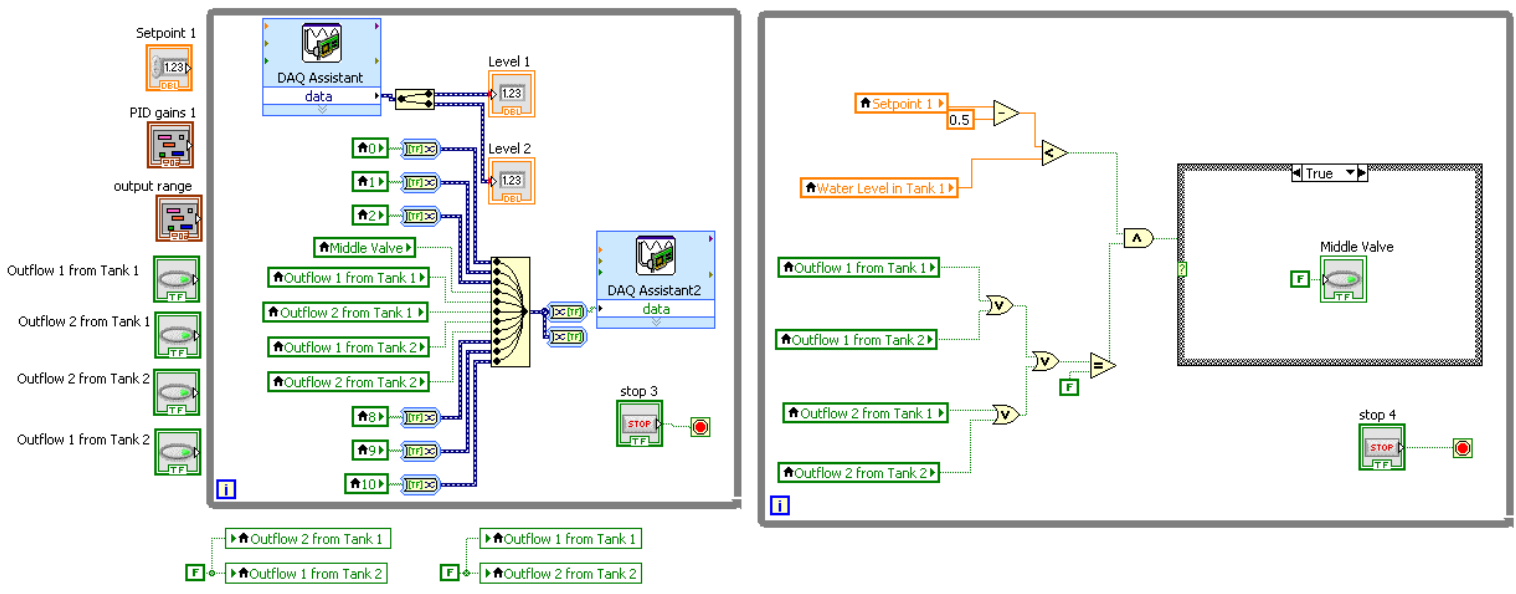

Fig.5 - Block diagram for outflow and middle valve operation

Signal has been sent to individual outflow valve as per required to ON/OFF to show the disturbances less or more via DAQ board.DAQ assistants have been placed to assist NI DAQ for outflow and coupled middle valve. In the right side block diagram, logic has been applied to OFF the middle valve automatically as desired water level is obtained in both the tanks and ON if not obtained. 


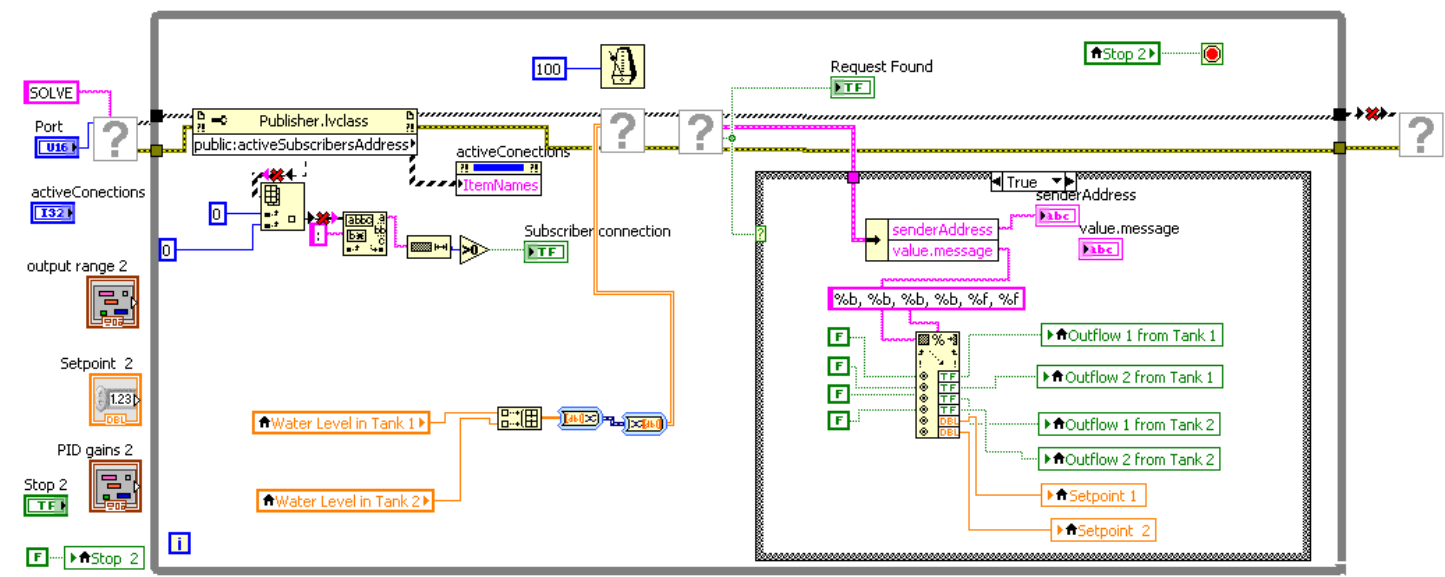

Fig. 6 - Block diagram for communication between subscriber and sender

In above communication block diagram, it has been shown that addresses for both publisher as well as sender has been placed and activated for subscriber so that subscriber can communicate with publisher easily whenever required. Subscriber has to make a request to conduct experiment to publisher then request is found and accepted and processed by publisher activated address. Subscriber also has the control on operational datas.

\section{References}

[1] Vishal Vasistha, "PID output fuzzified water level control in MIMO coupled tank system”, International Journal of Mechanical Engineering and Technology (IJMET), ISSN 0976 - 6340 (Print) ISSN 0976 - 6359 (Online) Volume 4, Issue 4, July - August (2013), pp. 138-153 C IAEME: www.iaeme.com/ijmet.asp, Journal Impact Factor (2013): 5.7731 (Calculated by GISI) www.jifactor.com

[2] http://sine.ni.com/nips/cds/view/p/lang/en/nid/210042 - SCCT Kit from NI

[3] www.daq-software.com/downloads/.../SCCT\%20User\%20Manual.pdf - SSCT tool kit user manual

[4] Muhammad Rehan, Fatima Tahir, Naeem Iqbal and Ghulam.," Modelling, Simulation and Decentralized Control of a Nonlinear Coupled Tank System", Department of Electrical Engineering, PIEAS, Second International Conference on Electrical Engineering University of Engineering and Technology, Lahore (Pakistan), 25-26 March 2008

[5] M. Khalid Khan, Sarah K. Spurgeon, " Robust MIMO water level control in interconnected twin-tanks using second order sliding mode control", Control and Instrumentation Group, Department of Engineering, University of Leicester, Leicester LE1 7RH, UK, 10 February 2005

[6] Qiang Xiong a, Wen-Jian Cai a, b, *, Mao-Jun He a, Equivalent transfer function method for PI/PID controller design of MIMO processes, Aug 28, 2012 